these parameters improved by $\geq 50 \%$. All patients improved according to the Paulus index. The reduction in C-reactive protein levels was particularly noteworthy, falling from a median of $39.5 \mathrm{mg} / \mathrm{L}$ at baseline to $8 \mathrm{mg} / \mathrm{L}$ by week 6 . Response was not affected by the treatment schedule.

\section{Repeated administration}

Retreatment of 8 responding patients from the initial study indicated that long-term disease suppression may be possible with regular $\mathrm{cA} 2$ therapy. ${ }^{[4]}$ Patients were treated with $\mathrm{cA} 210 \mathrm{mg} / \mathrm{kg}$ when disease relapsed. A response of similar magnitude to the initial response was seen after each treatment, but the duration of $20 \%$ response according to the Paulus criteria appeared to diminish with each successive dose. The duration of response was 9.1 weeks after the second treatment and 7.7 weeks after the 4th treatment in evaluable patients.

Although the antibody was still considered well tolerated, only 4 patients completed 4 cycles of treatment. One patient was withdrawn after experiencing vasovagal syncope after traumatic venepuncture, while 3 patients were withdrawn because of adverse events (urticaria, chronic sinusitis and development of antinuclear antibodies, respectively). A further patient experienced fever, headache and facial flushing. Human anti-cA2 antibody responses developed with repeated treatment in 4 patients.

\section{Efficacy confirmed in a placebo-controlled trial of $\mathbf{c A 2}$}

The efficacy observed in the open phase I/II study was confirmed in a placebo-controlled single-dose study. ${ }^{[2]} 73$ patients with refractory rheumatoid arthritis were randomised to receive a single infusion of placebo, cA2 $1 \mathrm{mg} / \mathrm{kg}$ or cA2 $10 \mathrm{mg} / \mathrm{kg}$.

The primary efficacy endpoint was defined as a $20 \%$ response at week 4 according to the Paulus index, which included assessment of tender joint and joint swelling scores, morning stiffness, patient and physician assessment of dis- ease severity, and ESR. According to these criteria, $79 \%$ of cA2 $10 \mathrm{mg} / \mathrm{kg}$ and $44 \%$ of cA $21 \mathrm{mg} / \mathrm{kg}$ recipients responded, compared with $8 \%$ of placebo recipients. In the high-dose cA 2 group, C-reactive protein levels were reduced from a mean of $64 \mathrm{mg} / \mathrm{L}$ at baseline to $35 \mathrm{mg} / \mathrm{L}$ at week 4 , with improvement seen as early as 24 hours after treatment.

Treatment with cA2 was generally well tolerated. Infections were the most common event, occurring in $5 \mathrm{cA} 2$ $1 \mathrm{mg} / \mathrm{kg}$ recipients and 1 patient in each of the other groups. Pneumonia precipitated the withdrawal of $1 \mathrm{cA} 2$ recipient from the trial.

\section{Open trial of CDP-571}

Celltech's CDP-571 is among the anti-TNF $\alpha$ antibodies under development by other drug companies. More than $40 \%$ of patients with severe arthritis reported a reduction in pain and a 22 to $27 \%$ lower average number of swollen and tender joints after a single dose of CDP-571 in a study of 36 patients. ${ }^{[5]}$ Even greater benefits were attained with repeated treatment after 8 weeks.

\section{References}

1. Brennan FM, Maini RN, Feldmann M. TNF $\alpha$ - a pivotal role in theumatoid arthritis? Br J Rheumatol 1992; 31: 293-8

2. Elliott MJ, Maini RN, Feldmann M, et al. Randomised double-blind comparison of chimeric monoclonal antibody to tumour necrosis factor $\alpha$ (cA2) versus placebo in rheumatoid arthritis. Lancet $1994 ; 344$ 1105-10

3. Elliott MJ, Maini RN, Feldmann M, et al. Treatment of rheumatoid arthritis with chimeric monoclonal antibodies to tumour necrosis factor $\alpha$. Arthritis Rheum 1993; 36: 1681-90

4. Elliott MJ, Maini RN, Feldmann M, et al. Repeated therapy with monoclonal antibody to tumour necrosis factor $\alpha$ (cA2) in patients with rheumatoid arthritis. Lancet 1994; 344: 1125-7

5. Celltech's antibody shows promise in trial as arthritis therapy. Wall St J 1994 Oct 26: B3

\title{
Addendum
}

\section{New information on HRT}

Encouraging data have been reported regarding the cardioprotective effects of hormone replacement therapy (HRT) in postmenopausal women. The results of the large Postmenopausal Estrogen/Progestin Interventions (PEPI) trial indicate that HRT regimens significantly improve the blood lipid profile compared with placebo, by increasing HDL cholesterol levels and reducing LDL cholesterol levels. ${ }^{[1]}$ Importantly, the differences between HRT and placebo were statistically significant irrespective of whether estrogen or estrogen combined with a progestogen was used for HRT. In addition, all HRT regimens prevented the ageassociated increase in plasma fibrinogen levels observed in placebo recipients, and none was associated with an increase in blood pressure.

Previously, there has been concern that the addition of a progestogen may negate the cardioprotective effects of estrogen monotherapy. In the PEPI trial, estrogen plus micronised progesterone had similar effects to estrogen alone, while the addition of medroxyprogesterone acetate

blunted the favourable effects of estrogen monotherapy on HDL cholesterol.

The article 'HRT is not to be taken lightly - pharmacoeconomics may assist prescribing decisions' [Drug Ther Perspect 1994 Nov 28; 4 (11): 1-4] drew attention to the profound pharmacoeconomic effect of various assumptions about the cardiovascular benefit of HRT. The PEPI trial results suggest that some of the more optimistic assumptions, in which both estrogen monotherapy and combined estrogen + progestogen regimens are considered to be cardioprotective, may apply. These results are of particular relevance to women with intact uteri, since estrogen monotherapy places them at risk of endometrial hyperplasia and cancer.

However, the PEPI trial looked at risk factors for cardiovascular disease rather than actual clinical endpoints, and so further evidence is required before the true pharmacoeconomic effects of HRT in postmenopausal women may be assessed.

\section{Reference}

1. The Writing Group for the PEPI trial. Effects of estrogen or estrogen/progestin regimens on heart disease risk factors in postmenopausal women. JAMA 1994; 273: 199-208 